\title{
Measuring the trilinear neutral Higgs boson couplings in the MSSM at $e^{+} e^{-}$colliders
}

\section{Charanjit K. Khosa*}

Centre for High Energy Physics, Indian Institute of Science, Bangalore 560 012, India

E-mail: khosacharanjit@gmail.com

\section{P. N. Pandita}

Centre for High Energy Physics, Indian Institute of Science, Bangalore 560 012, India

E-mail: panditalassociates.iucaa.in

\begin{abstract}
We consider the measurement of the trilinear couplings of the neutral Higgs bosons $\left(H^{0}, h^{0}\right)$ in the minimal supersymmetric standard model (MSSM) at a high energy $e^{+} e^{-}$linear collider in the light of the discovery of a Higgs boson at the CERN Large Hadron Collider (LHC). We identify the state observed at the LHC with the lightest CP-even Higgs boson of the MSSM. We implement this constraint, as well as all the other relevant experimental constraints, on the parameter space of the MSSM in order to study the feasibility of measuring the trilinear couplings of the neutral Higgs bosons. For the measurement of trilinear couplings, we consider the multiple Higgs production processes. We delineate the regions of MSSM parameter space where the trilinear couplings of the neutral Higgs bosons could be measured at a high energy electron-positron collider.
\end{abstract}

38th International Conference on High Energy Physics 3-10 August 2016

Chicago, USA

*Speaker. 


\section{Introduction}

The ATLAS and CMS experiments at the CERN Large Hadron Collider (LHC) have discovered a particle with mass about $125 \mathrm{GeV}$ [1] whose properties are consistent with the Higgs boson of the standard model (SM). To confirm that this particle is the Higgs boson of the SM, one must measure its couplings to other particles as well as to itself. In the SM, the potential of the Higgs doublet $\phi$ which breaks the $S U(2) \times U(1)$ gauge symmetry is written as

$$
V_{S M}^{h}=\lambda\left(\phi^{2}-\frac{v^{2}}{2}\right)^{2}=\frac{m_{h}^{2}}{2} h^{2}+\lambda_{h h h}^{S M} \frac{h^{3}}{3 !}+\lambda_{h h h h}^{S M} \frac{h^{4}}{4 !} \quad ; \quad \phi=\frac{1}{\sqrt{2}}\left(\begin{array}{ll}
0 & v+h
\end{array}\right)^{T},
$$

where $h$ is the physical Higgs boson, and $\lambda_{h h h}^{S M}=3 m_{h}^{2} / m_{Z}^{2}=5.6454$ (in units of $\left(\sqrt{2} G_{F}\right)^{1 / 2} m_{Z}^{2}=$ $33.77 \mathrm{GeV}$ ) and $\lambda_{h h h h}^{S M}=3 m_{h}^{2} / m_{Z}^{4}=0.00068 \mathrm{GeV}^{-2}$ (in units of $\left(\sqrt{2} G_{F}\right) m_{Z}^{4}=1140.52 \mathrm{GeV}^{2}$ ) are the triple and quartic Higgs couplings, respectively. One must, therefore, measure these couplings in order to confirm the idea of the spontaneous breaking of the underlying gauge symmetry. Any deviation from the SM prediction for these couplings will signal the existence of new physics beyond SM. Supersymmetry (SUSY)[2] is the leading candidate for the new physics which stabilizes the Higgs mass against large radiative corrections. The supersymmetric version of the SM, known as minimal supersymmetric standard model (MSSM)[2] not only solves the naturalness problem of the SM, but also leads to gauge coupling unification at a large scale $\left(M_{\mathrm{GUT}}=2 \times 10^{16} \mathrm{GeV}\right)$. Furthermore, the lightest supersymmetric particle, usually the lightest neutralino, could be a possible dark matter candidate in $R$ - parity conserving models. In the MSSM, there are two Higgs doublets $\left(H_{2}, H_{1}\right.$, ) with opposite hypercharge, which break the SM gauge symmetry, and there are five physical Higgs bosons $\left(h^{0}, H^{0}, A^{0}, H^{ \pm}\right)$. In this paper we address the question of the measurement of some of the trilinear couplings of the neutral Higgs bosons $h^{0}, H^{0}$ at a high energy electron-positron $\left(e^{+} e^{-}\right)$collider.

\section{MSSM Higgs sector and trilinear couplings of $h^{0}, H^{0}$}

In this section we shall consider the trilinear couplings of the CP-even Higgs bosons $\left(h^{0}, H^{0}\right)$. At the tree level the masses of all the Higgs bosons and their couplings can be written in terms of the mass of the CP-odd Higgs boson $m_{A^{0}}$ and $\tan \beta=<H_{2}^{0}>/<H_{1}^{0}>$. For the phenomenological survey, the Higgs sector of the MSSM can be divided into two regions known as non-decoupling regime and decoupling regime. In the first case, $m_{A^{0}} \leq 130 \mathrm{GeV}$, and heavy Higgs $\left(H^{0}\right)$ is considered as SM-like (observed) state. On the other hand, decoupling regime is represented by $m_{A^{0}}>$ $300 \mathrm{GeV}$, and the light Higgs boson $\left(h^{0}\right)$ is identified with the observed $125 \mathrm{GeV}$ Higgs boson. We shall here focus on the decoupling scenario. The mass matrix for the CP-even Higgs bosons of the MSSM can be written as

$$
\mathscr{M}^{2}=\left[\begin{array}{cc}
m_{A^{0}}^{2} \sin ^{2} \beta+m_{Z}^{2} \cos ^{2} \beta & -\left(m_{Z}^{2}+m_{A^{0}}^{2}\right) \sin \beta \cos \beta \\
-\left(m_{Z}^{2}+m_{A^{0}}^{2}\right) \sin \beta \cos \beta & m_{A^{0}}^{2} \cos ^{2} \beta+m_{Z}^{2} \sin ^{2} \beta
\end{array}\right]+\frac{3 g^{2}}{16 \pi^{2} m_{W}^{2}}\left[\begin{array}{cc}
\Delta_{11} \Delta_{12} \\
\Delta_{12} \Delta_{22}
\end{array}\right],
$$

where the radiative corrections $\Delta_{i j}$ are sensitive, besides other parameters, to the top-stop masses. Other parameters that enter the calculations are the the Higgs(ino) parameter $\mu$, and $\tan \beta$. We have used CalcHEP [3] for the numerical calculations of the Higgs spectrum. Furthermore, we have adjusted the trilinear parameter $A_{t}$ so as to fix the mass of the lightest Higgs $\left(m_{h^{0}}\right)$ in the range $122-128 \mathrm{GeV}$. In Fig. 1 (left panel) we show the variation of the CP-even heavy Higgs mass $\left(m_{H^{0}}\right)$ as a function of the parameter $\mu$ for fixed values of the supersymmetry breaking scale $\left(M_{S}\right)$ and 
$\tan \beta$. We see from this Fig. that the heavy Higgs mass is weakly dependent on $M_{S}$ for fixed value of $\tan \beta$. Now coming to the Higgs self couplings, assuming $C P$-conservation, we have six couplings amongst the neutral Higgs bosons of the MSSM. These are written as $\lambda_{\text {hhh }}, \lambda_{H h h}, \lambda_{h A A}, \lambda_{H A A}$, $\lambda_{H H H}$ and $\lambda_{H H h}$. As in the case of the masses of the Higgs bosons, these trilinear couplings also obtain significant radiative corrections [4]. We can, then, write these trilinear couplings generically as $\lambda=\lambda^{0}+\Delta \lambda$ where $\lambda^{0}$ is the tree-level coupling and $\Delta \lambda$ are the radiative correction. In this study we shall consider only two of these trilinear couplings, namely (in units of $\left(\sqrt{2} G_{F}\right)^{1 / 2} m_{Z}^{2}$ ) $\lambda_{\text {hhh }}^{0}=3 \cos 2 \alpha \sin (\beta+\alpha)$ and $\lambda_{\text {Hhh }}^{0}=2 \sin 2 \alpha \sin (\beta+\alpha)-\cos 2 \alpha \cos (\beta+\alpha)$, where $\alpha$ is the mixing angle in the CP-even Higgs sector. These couplings are sensitive to $m_{A^{0}}$ values of upto $500 \mathrm{GeV}$. The dependence of the trilinear couplings on the parameter $\mu$ is shown in Fig.1.
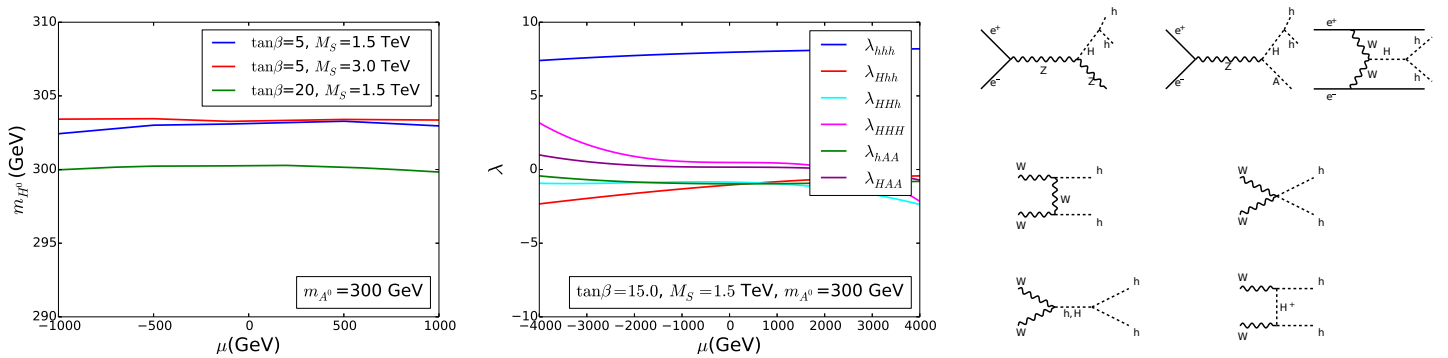

Figure 1: The mass of heavier Higgs boson $m_{H^{0}}$ as a function of $\mu$ parameter for fixed $\tan \beta$ and $M_{S}$ (left panel); variation of radiatively corrected trilinear couplings in MSSM with $\mu$ parameter(central panel); Feynman diagrams for resonant production of $h h$, through $e^{+} e^{-} \rightarrow H Z, H A, v_{e} \bar{v}_{e} H$ (where $H \rightarrow h h$ in the final state) and through non-resonant $W W$ fusion (right panel).

\section{Measurement of trilinear couplings}

We now discuss the multiple Higgs production processes which can be used to study the trilinear couplings involving $H^{0}$ and $h^{0}$. We consider the production of heavy Higgs boson through Higgs-strahlung $e^{+} e^{-} \rightarrow Z H$, associated production with CP-odd Higgs boson $e^{+} e^{-} \rightarrow A H$, and $W W$ fusion mechanism $e^{+} e^{-} \rightarrow v_{e} \bar{v}_{e} H$ (see Fig. 1 (right panel) for Feynman diagrams and [4] for cross-sections). Multiple light Higgs bosons $\left(h^{0}\right)$ can be produced through heavy CP-even Higgs boson decay. Non- resonant $h h$ pair could also be produced with $Z$ or $A$ [4]. Associated production of $A$ with $h$, where $A$ decays to $h Z$, contributes as the background to the multiple Higgs production processes. In Fig. 2, left panel, we show the cross-section for $e^{+} e^{-} \rightarrow Z H, A H, H v \bar{v}$ as a func-
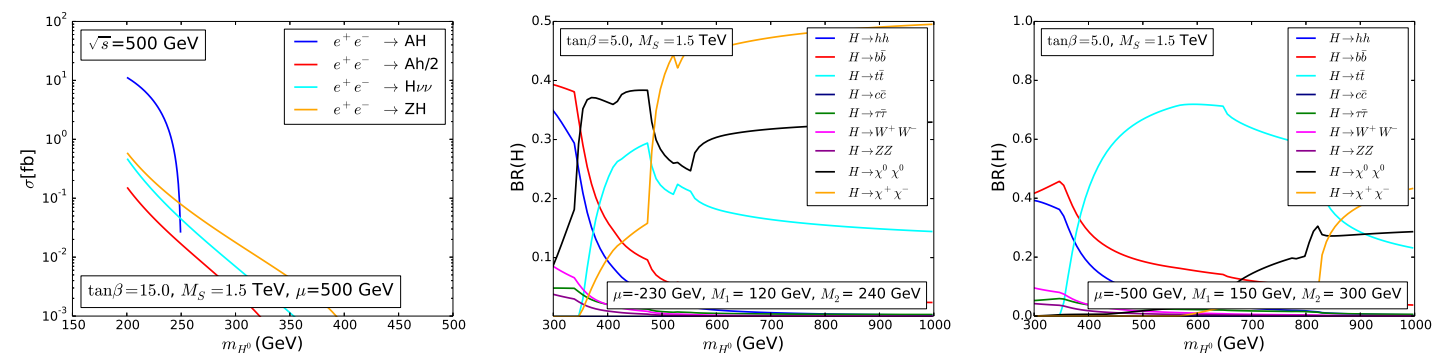

Figure 2: Cross-section for $e^{+} e^{-} \rightarrow A H, Z H, A h / 2, H v \bar{v}$ as the function of $m_{H^{0}}$ for $\sqrt{s}=500 \mathrm{GeV}$ (left); the branching fractions for different decay channels of $H$ for both the benchmark points(central and right).

tion of $m_{H^{0}}$ for $\sqrt{s}=500 \mathrm{GeV}$. We choose two benchmark values for the parameters $\left(\mu, M_{1}, M_{2}\right)$ $=(-230,120,240)$ and $(-500,150,300)$, respectively, so as to include SUSY particles in the final 
state of the heavy Higgs boson decay. In Fig. 2, central and right panel, we show the branching fractions of heavy Higgs decay into different channels. Second benchmark values have larger $\mathrm{BR}(H \rightarrow h h)$ because neutralino and chargino spectrum is heavy as compared to the first benchmark point. Consequently, $\operatorname{BR}\left(H \rightarrow \chi^{0} \chi^{0}, \chi^{+} \chi^{-}\right)$is suppressed. In Fig. 3, for the measurement of $\lambda_{H h h}$ coupling, we show the contours of $\sigma(H) \times \mathrm{BR}(H \rightarrow h h)$ for $\sqrt{s}=500 \mathrm{GeV}$ and $\sqrt{s}=$ $1.5 \mathrm{TeV}$, respectively for the first benchmark point. The decay $H \rightarrow h h$ is kinematically forbidden for $m_{A^{0}} \approx m_{H^{0}} \leq 250 \mathrm{GeV}$, and this branching ratio decreases as we move diagonally upward in the $\left(m_{A^{0}}, \tan \beta\right)$ plane. The lower left corner of $\left(m_{A^{0}}, \tan \beta\right)$ plane is the suitable region to measure $\lambda_{H h h}$ coupling. The non-resonant WW fusion to $h h v_{e} \bar{v}_{e}$ final state involves both $\lambda_{h h h}$ and $\lambda_{H h h}$ couplings. Having an estimate of $\lambda_{H h h}$, we use this process to measure $\lambda_{h h h}$ coupling. We can see from the Fig. 3, right panel, that the $\sigma\left(h h v_{e} \bar{v}_{e}\right)$ is almost independent of $m_{A^{0}}$ and $\tan \beta$.
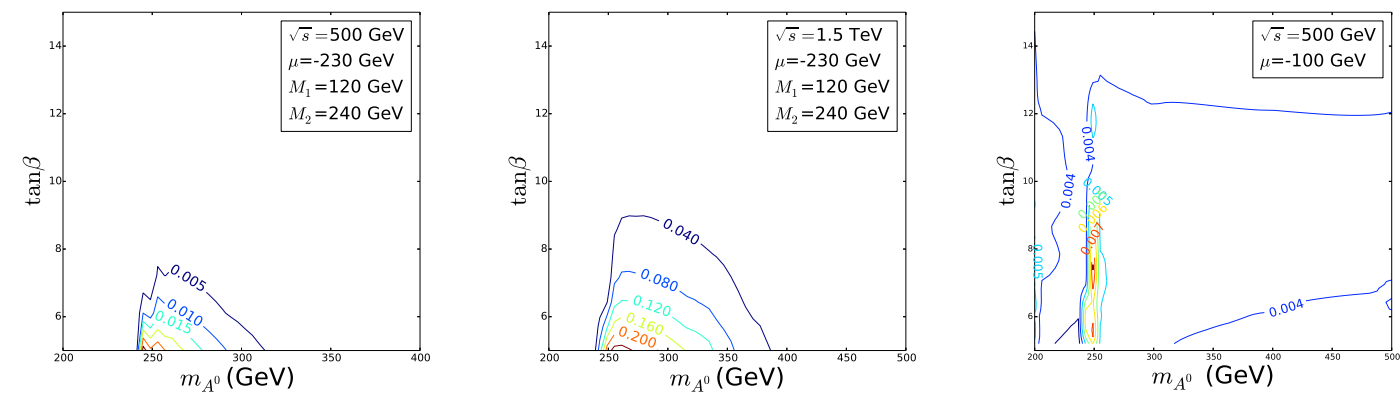

Figure 3: The contours of constant $\sigma(H) \times B R(H \rightarrow h h$ ) (in fb) (left and central panel); the contours of non-resonant $\sigma(e e \rightarrow h h v \bar{v})$ (in fb) via non-resonant WW fusion (right).

\section{Conclusions}

We have studied the trilinear couplings of the CP-even Higgs bosons $\left(H^{0}, h^{0}\right)$ in the minimal supersymmetric standard model, and their measurement at a high energy $e^{+} e^{-}$collider. In doing so we have identified the resonance observed at the CERN LHC at $125 \mathrm{GeV}$ with the lightest CP even Higgs boson of the MSSM. By estimating the production cross-section of the various processes involving multiple Higgs bosons, we have delineated the regions in the $\left(m_{A^{0}}, \tan \beta\right)$ space where trilinear couplings $\lambda_{H h h}$ and $\lambda_{h h h}$ can be measured at an $e^{+} e^{-}$collider. For the coupling $\lambda_{H h h}$, we have $\sigma(H) \times B R(H \rightarrow h h) \approx 0.005 \mathrm{fb}$ and $0.04 \mathrm{fb}$ for $\sqrt{s}=500 \mathrm{GeV}$ and $\sqrt{s}=1.5 \mathrm{TeV}$, respectively, and it decreases along the diagonal upward direction in the $\left(m_{A^{0}}, \tan \beta\right)$ plane. We estimate the coupling $\lambda_{h h h}$ through non-resonant WW fusion process. Precise knowledge of neutralino and chargino masses is crucial in order to determine the trilinear couplings of $H^{0}, h^{0}$ of the MSSM.

\section{References}

[1] G. Aad et al. [ATLAS and CMS Collaborations], Combined Measurement of the Higgs Boson Mass in pp Collisions at $\sqrt{s}=7$ and $8 \mathrm{TeV}$ with the ATLAS and CMS Experiments, Phys. Rev. Lett. 114 191803 (2015).

[2] H. P. Nilles, Supersymmetry, Supergravity and Particle Physics, Phys. Rept. 1101 (1984).

[3] A. Belyaev, N. D. Christensen and A. Pukhov, CalcHEP 3.4 for collider physics within and beyond the Standard Model, Comput. Phys. Commun. 1841729 (2013).

[4] P. Osland and P. N. Pandita, Measuring the trilinear couplings of MSSM neutral Higgs bosons at high-energy e $e^{+} e^{-}$colliders, Phys. Rev. D 59055013 (1999); [hep-ph/9911295].

[5] C. K. Khosa and P. N. Pandita, Measuring the trilinear neutral Higgs boson couplings in the minimal supersymmetric standard model at $e^{+} e^{-}$colliders in the light of the discovery of a Higgs boson, Int. J. Mod. Phys. A 311650108 (2016), and references therein. 